SESSION 6: Spatially-resolved analyses of $\mathrm{z}>2$ galaxies 


\title{
Resolved views on early galaxy evolution
}

\author{
Stijn Wuyts ${ }^{1}$ (D) and Natascha M. Förster Schreiber ${ }^{2}$ \\ ${ }^{1}$ Dept. of Physics, University of Bath, Claverton Down, Bath, BA2 7AY, UK \\ email: s.wuyts@bath.ac.uk \\ ${ }^{2}$ Max-Planck-Institut für extraterrestrische Physik, Giessenbachstr. 1, \\ Garching, D-85737, Germany \\ email: forster@mpe.mpg.de
}

\begin{abstract}
Resolved observations of star-forming galaxies at cosmic noon with the Hubble Space Telescope and large ground-based facilities provide a view on the spatial distribution of stars, gas and dust, and probe gaseous motions revealing the central gravitational potential and local feedback processes at play. In this paper, we review recent insights gained from such observations, with an emphasis on results obtained through optical/near-infrared imaging and imaging spectroscopy. Their context and implications are documented more fully in a forthcoming review article by Förster Schreiber \& Wuyts (in prep).
\end{abstract}

Keywords. Galaxies: structure, Galaxies: kinematics, Galaxies: high-redshift

\section{Introduction}

Census. Accumulating over more than a decade, a large cross section of the galaxy evolution community has contributed to assembling a wedding cake of deep legacy fields from which consistent multi-wavelength photometric catalogs have been extracted and made publicly available. Ranging from ultra-deep pencil-beam surveys such as the Hubble XDF to areas of $2 \mathrm{deg}^{2}$ (COSMOS) and beyond (e.g., Hyper-SuprimeCam, VIDEO, KIDS+Viking) they complement each other in a balancing of area versus depth.

Another dimension along which legacy imaging surveys have advanced and augmented the potential to infer physical properties of galaxies is wavelength. The spectral coverage in the aforementioned legacy deep fields now routinely extends from X-ray diagnostics of AGN activity to a bolometric census of star formation by pairing the rest-UV emission as observed with HST with far infrared constraints on the amount of obscured and dust-reprocessed emission from young stars as observed with Spitzer/MIPS and Herschel. Importantly, it also encompasses increasingly a finer sampling of galaxy Spectral Energy Distributions (SEDs) through the use of medium-band filters (e.g., zFOURGE) and slitless grism spectroscopy (e.g., 3D-HST). The intermediate $R \sim 13-130$ SED sampling these surveys provide enables a sensitive improvement in photometric redshift estimates, which continue to be of great importance as high-resolution spectroscopic follow-up campaigns struggle to reach high levels of completeness over the same dimensions in area and depth. The enhancement in redshift quality can further aid spectroscopic follow-up campaigns, for example by allowing to select specifically those targets that have their emission lines of interest redshifted away from $\mathrm{OH}$ sky lines that render significant swathes of the near-infrared wavelength regime unsuitable for detailed line profile analyses as discussed in Section 3 (Wisnioski et al. 2015). Finally, by more accurately pinpointing the location and strength of continuum breaks they yield improved constraints on the mass-to-light ratios of distant galaxies and the stellar population (age) or dust (attenuation) properties responsible for it. 
It is this richness of multi-wavelength data that has facilitated a mass-complete census of star-forming and quiescent galaxies over more than $90 \%$ of cosmic history (e.g., Davidzon et al. 2017).

Scaling relations. Alongside an improved census of the number of galaxies as a function of mass, redshift and type (star-forming/quiescent) multi-wavelength lookback surveys have helped establish the regularity of galaxy properties across cosmic time. This regularity is captured by a set of scaling relations aimed at describing the star-forming galaxy (SFG) population as a one-parameter family. At any given epoch, physical properties of SFGs such as their star formation rate (SFR), gas content, metallicity, rotational velocity and size can be parameterized as a function of galaxy stellar mass. The change in zero point, slope and/or shape of such scaling relations then encodes their evolution in a population averaged sense (Speagle et al. 2014; Tacconi et al. 2018; Sanders et al. 2018; Übler et al. 2017; van der Wel et al. 2014).

Evolution. The evolution of population-averaged physical properties evaluated at fixed mass does not equate to the evolution of individual galaxies, which grow in mass over time. In recent years, considerable efforts have therefore gone into reconstructing the growth history of individual systems. Perhaps the most common approach of connecting progenitor and descendant galaxies across cosmic time consists of assuming that their relative mass ranking remains preserved (i.e., the most massive galaxy today was also the most massive at earlier epochs and so on), in which case progenitors and descendants can be considered to live at the same comoving number density. Curves of stellar mass growth as a function of redshift can then be combined with the aforementioned scaling relations to establish how an individual galaxy consumes its gas, enriches its ISM, grows its size and builds up angular momentum.

Caveats to this methodology apply. Modifications to the prescription of constructing progenitor-descendant sequences have been proposed by Torrey et al. (2017) on the basis of empirical galaxy stellar mass functions and an assessment of the significant galaxy-togalaxy scatter in growth rates seen in cosmological simulations, even in the absence of frequent major mergers. The primary notion that SFGs can be treated as a one-parameter family can also be questioned. On the grounds of cosmological simulations, Matthee \& Schaye (2019) argued that the scatter around the star-forming main sequence features contributions both from short-term stochasticity (so-called 'breathing' due to the cycle of star formation feedback) and a long-term differentiation in growth histories tied to halo formation times. Along similar lines, taking a more empirical view at the evolving galaxy population, Abramson et al. (2016) describe the main sequence and size-mass scaling relations as emerging from a population moving through them rather than being composed of the same systems at all times.

Despite the above caveats which prompt further study in this area, specifically regarding the physical origin of scatter around scaling relations, a few basic conclusions can be drawn from the above line of reasoning. For a galaxy of Milky Way mass today, it reveals the so-called era of cosmic noon $(1<z<3)$ as a key epoch during which the star formation activity peaked, the gas richness dropped most dramatically (despite continuing yet declining gaseous accretion onto the galaxy) and the chemical enrichment proceeded most quickly. In relative terms, the rate of size growth was maintained over a much more extended period of time down to the present day (see Figure 1 of Förster Schreiber \& Wuyts in prep).

Toward a multi-wavelength resolved view of galaxies at cosmic noon. While highlighting the importance of the cosmic noon era to understand the growth history of Milky Way and higher mass galaxies, the above galaxy-integrated quantities need to be paired with a resolved view of their structure and kinematics if we are to understand the physical drivers behind their evolution. In fact, as we illustrate below, it is a multi-wavelength resolved 


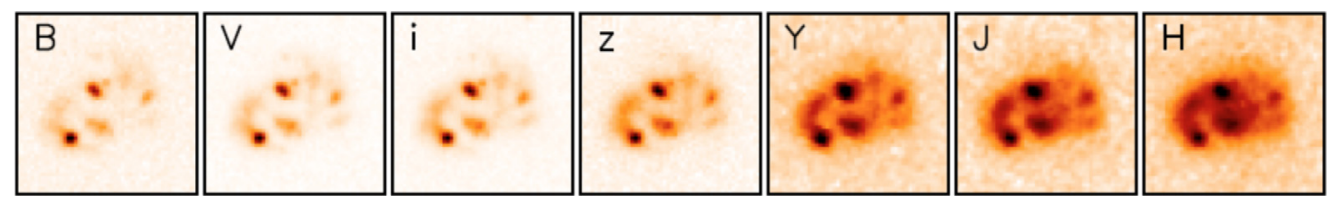

Figure 1. Example of a $z=2.13$ main sequence galaxy in the HUDF as imaged in the F435W, F606W, F775W, F850LP, F105W, F125W and F160W bands. Postage stamps measure $3^{\prime \prime} \times 3^{\prime \prime}$. This galaxy features a clumpy structure that is most prominent in the rest-frame UV and becomes progressively less pronounced toward redder wavelengths.

view that is needed to construct a comprehensive picture of the physical processes at play. With multi-band resolved maps of thousands of distant galaxies in the rest-UV to optical, resolved observations of ionized gas line emission in hundreds of them (thousands if relying on stacking of low-S $/ \mathrm{N} \mathrm{H} \alpha$ maps from HST grism spectroscopy out to $z<1.5$ ), and far-infrared continuum and molecular gas emission line maps for dozens, the field is increasingly moving towards such spatially resolved, multi-tracer analyses. That said, the number of normal SFGs for which the full complement of multi-wavelength diagnostics is available and sensitively mapped down to kiloparsec scales remains modest. This is in part due to AO-assisted Integral Field Unit (IFU) instrumentation (e.g., SINFONI and its upgraded incarnation ERIS) lacking the multiplexing capabilities of seeing limited IFU instruments such as KMOS, and the emphasis on bright sources during the early cycles of ALMA observations.

In this paper, we review key lessons learned from high-resolution imaging campaigns, starting with monochromatic measures of galaxy structure before delving into a multiwavelength view (Section 2). We next touch upon a number of insights gained from imaging spectroscopy, with dynamics offering a probe of the gravitational potential and non-gravitational motions complementing metallicity gradients as diagnostics of the feedback processes at play (Section 3). Finally, we present an outlook to new instrumentation and facilities projected to come on-line in the next decade and enabling the tackling of a number of remaining open questions (Section 4).

\section{Lessons from high-resolution imaging}

Monochromatic structure. Deep HST/WFC3 imaging of the CANDELS fields and more recent wide-area extensions over as much as 0.6 square degrees with COSMOS-DASH provide a means to characterize the rest-optical structure of galaxies spanning a large dynamic range in mass out to cosmic noon. Analyzing the 0th order structural measure of size (parameterized as the half-light radius of a single Sersic model fit to the 2D surface brightness distribution), van der Wel et al. (2014) characterize the size growth of SFGs at fixed mass to proceed as $\propto(1+z)^{-0.75}$ and of quiescent galaxies as $\propto(1+z)^{-1.48}$, with no appreciable evolution in the slope of the size - mass relation for either of the two types. Parameterized as a function of the Hubble parameter $\left(R_{e}(z) \propto H(z)^{-2 / 3}\right)$, the size growth at fixed mass is consistent with the halo size evolution at fixed halo mass, validating a basic assumption underpinning virtually all semi-analytical models for galaxy formation, namely that the baryons accreted onto a galaxy disk inherit the specific angular momentum of their dark matter haloes (Mo, Mao \& White (1998)). A follow-up analysis by Huang et al. (2017) of the galaxy size - halo size relation, inferred from tying the empirical size - mass relation with abundance matching results, arrives at similar conclusions, which are also echoed by direct, kinematic measurements of the angular momentum distribution of galaxies out to $z \sim 2.5$ (Burkert et al. 2016). In the same Mo, Mao \& White (1998) formalism for disk formation, the distribution of halo spin parameters found in dark matter simulations is by itself sufficient to account for 
the observed scatter in galaxy sizes at a given mass (Bullock et al. 2001; Kravtsov 2013) and has more recently even been argued to be too large, requiring an alteration of disk growth in extreme spin parameter halos or an entirely different formalism altogether (Zanisi et al. 2019).

In passing, we note that the above conclusions reflect on the full, mass-complete population of SFGs (and quiescent galaxies). Individual sub-populations of SFGs, such as Lyman Break Galaxies (LBGs), are known to feature different (in the case of LBGs faster) size evolution (e.g., Allen et al. 2017). Moreover, as one considers higher redshift SFGs it is well established that their appearance becomes less regular, raising questions about the metric used to quantify size. Ribeiro et al. (2016) for example argue that no size evolution is apparent for SFGs at $2<z<4.5$ when a non-parametric count of pixels above a given surface brightness threshold is used as the basis for measuring sizes, rather than fitting of a smooth Sersic model.

Even in the context of Sersic fitting, the half-light radius of a galaxy does not capture its structure fully. A 1st order additional specification considers the profile shape, as parameterized by the Sersic index $n$. It is well known that at all times star-forming and quiescent galaxies differ in their Sersic index distributions, with quiescent galaxies featuring cuspier, more centrally concentrated surface brightness distributions (e.g., Wuyts et al. 2011; Whitaker et al. 2015). As a consequence, the relative distribution of SFGs and quiescent galaxies in the size - mass plane is altered when defining galaxy size based on an aperture containing a different percentage than $50 \%$ of the light. This is explored in more depth by Mowla et al. (2019), who leverage the improved number statistics at the massive end provided by COSMOS-DASH, and find SFGs and quiescent galaxies to occupy the same size - mass relation when adopting $R_{80}$ (the radius within which $80 \%$ of the light is enclosed) as size definition. They argue it is this size, comprising the bulk of the stars, that relates most tightly to the virial radius of the parent halo $\left(R_{80}=0.047 R_{\mathrm{vir}}\right)$, for both galaxy types and across mass and redshift.

Panchromatic structure: rest-UV to rest-optical broad-band colors. With HST legacy surveys such as CANDELS providing up to 7 bands of ACS+WFC3 imaging, sampling the rest-UV to rest-optical SEDs of galaxies at cosmic noon, it was soon realised that galaxies feature internal color variations, encoding spatial variations in the mass-to-light $(M / L)$ ratio (see, e.g., Figure 1$)$.

UV-selected star-forming clumps feature less prominently at longer wavelengths and largely filter out as regions of reduced $M / L$ when reconstructing the stellar mass distributions, which tend to be more centrally concentrated (Wuyts et al. 2012). Even in the rest-UV, it should be noted that the underlying diffuse component, and not the giant star-forming clumps, contribute the bulk of the blue light emitted by SFGs at cosmic noon. Taking the census over a mass-complete sample of galaxies at $0.5<z<2.5$ and focusing on the azimuthally averaged trends, Figure 2 presents the strength of radial color variations as a function of mass, redshift and galaxy type. Here, we parameterize this in terms of the so-called red centeredness: the difference between an inner and outer rest-optical $(U-V)_{\text {rest }}$ color, as interpolated using EAZY (Brammer et al. 2008) from the resolved 7-band imaging in the CANDELS-GOODS fields. The boundary between inner and outer regions is taken to be $2 \mathrm{kpc}$, but similar trends are observed when evaluating red centeredness calculated from the color within and outside the galaxies' half-stellar mass radii. It is apparent that star-forming galaxies feature a more pronounced redcenteredness, especially toward higher masses and increasingly so at later times. While any mass or redshift dependence is reduced for quiescent galaxies, it is clear that they too feature redder centers than outskirts (i.e., $\left.(U-V)_{\text {in }}-(U-V)_{\text {out }}>0\right)$, in line with predictions from simulations by Wuyts et al. (2010) where these trends come about through a superposition of radial age, metallicity and dust extinction gradients. 


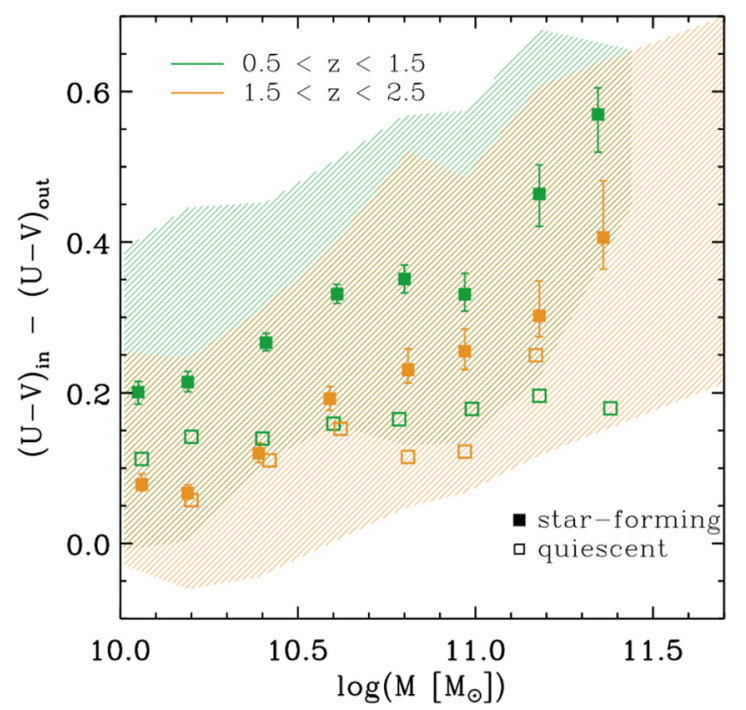

Figure 2. Red centeredness of a mass-complete sample of $M_{\text {star }}>10^{10} M_{\odot}$ galaxies extracted from the CANDELS-GOODS fields. SFGs and quiescent galaxies both feature negative color gradients, with their inner rest-optical colors being redder than those measured outside a fixed aperture of $2 \mathrm{kpc}$. The red centeredness of SFGs increases progressively toward higher masses, and is more enhanced at $z \sim 1$ than at $z \sim 2$. Similar trends are found when defining inner/outer colors within/outside an aperture that scales with galaxy size. Quiescent galaxies are also red centered, but exhibit a weaker mass and redshift dependence.

Translated to stellar mass maps, the inferred spatially resolved color profiles imply that SFGs build up significant stellar bulges, comprising over $40 \%$ of the total stellar mass, as they grow beyond the Schechter mass, already prior to their eventual quenching (Lang et al. 2014). The implications for the (shallower) size - mass relation, quantified on stellar mass rather than light distributions, were recently presented by Suess et al. (2019).

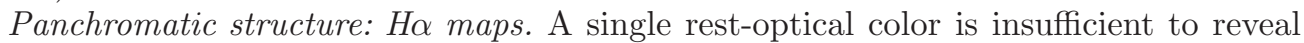
the origin of the observed spatial $M / L$ ratio variations, as age tracks from stellar population models and dust vectors are closely aligned along a similar $M / L-(U-V)_{\text {rest }}$ relation. Owing to HST slitless grism spectroscopy, near-IR IFU and far-IR interferometric observations from the ground, other tracers can increasingly be tied into the analysis of resolved stellar populations and dust distributions. At present, $\mathrm{H} \alpha$ maps on kiloparsec scales are available for statistical stacking analyses for several thousands of galaxies from 3D-HST (Nelson et al. 2012, 2013, 2016a; Wuyts et al. 2013). For several dozen individual cases the $\mathrm{H} \alpha$ surface brightness distribution has been mapped at the same resolution to a greater sensitivity (and without blended [NII] emission) using adaptive optics assisted IFU spectroscopy (Förster Schreiber et al. 2018). More recently, Wilman et al. (2019) demonstrated that basic $\mathrm{H} \alpha$ size measurements can also be recovered from seeing-limited IFU observations. The multiplexing capabilities of KMOS on VLT allow for hundreds of such individual size measurements. In common between these studies is the finding that the $\mathrm{H} \alpha$ emission traces an exponential disk distribution, with a scale length that exceeds that of the rest-optical continuum by a factor $\sim 1.2$. This implies a centrally depressed $\mathrm{H} \alpha$ equivalent width (EW), with the slope of the $\mathrm{H} \alpha \mathrm{EW}$ profiles getting steeper as one considers more massive galaxies.

While it is tempting to interpret the $\mathrm{H} \alpha \mathrm{EW}$ profiles as probing variations in the local star formation activity $\left(s S F R \equiv S F R / M_{\text {star }}\right)$ and the observed trends therefore as 
being in line with expectations from inside-out growth and/or quenching scenarios, it is important to note that dust extinction can also affect the observed $\mathrm{H} \alpha \mathrm{EW}$. This is specifically the case as the galaxy's nebular emission may suffer from enhanced levels of attenuation compared to the underlying stellar continuum, due to dust embedded in the birth clouds from which the ionized gas line emission emerges. Various methods have been pursued to characterize the presence of spatial variations, and particularly radial gradients, in dust attenuation within distant SFGs: using HST broad-band colors (e.g., spaxel-by-spaxel SED modeling by Wuyts et al. 2012; rest-frame UVI color-color diagrams by Liu et al. (2017); rest-UV slopes $\beta$ by Tacchella et al. 2018) or more directly using the Balmer decrement (Nelson et al. 2016b, albeit based on stacking of galaxies within relatively large mass bins). All methods agree on finding significant negative gradients, commonly reaching 1.5 to 2 magnitudes of attenuation in the centers of massive galaxies. Quantitative differences in the inferred slopes are seen, which may in part be attributed to differences in sample selection, but more likely also stem from the different methodologies employed. Propagating the estimated dust corrections to quantify the radial sSFR profiles of SFGs at $z \sim 2$, Tacchella et al. (2018) conclude that they are generally flat, implying an even build-up of stellar mass by star formation across all radii, with the exception of the most massive objects $\left(M_{\text {star }}>10^{11} M_{\odot}\right)$ where a central depression in the (dustcorrected) $\mathrm{H} \alpha \mathrm{EW}$ remains apparent.

Panchromatic structure: dust continuum maps. At the same time, new insights on the nature of the most massive $\left(M_{\text {star }}>10^{11} M_{\odot}\right) z \sim 2$ SFGs are emerging from ALMA. These offer a more bolometric view on the (dust-reprocessed) emission from young stars. Carrying out observations at $870 \mu \mathrm{m}$ in a compact and extended configuration, Tadaki et al. (2017) recovers dust continuum sizes for 12 massive galaxies, nine of which are associated with extremely compact dust emission less than $1.5 \mathrm{kpc}$ in size. Such sizes are more than a factor of 2 smaller than the characteristic rest-optical sizes. Exploiting a purely stellar mass selected sample that is 6 times as large, Tadaki et al. (in prep) place these results on a more robust statistical footing. Barring gradients in dust temperature, which could be revealed by multi-band ALMA observations, the compact dust continuum sizes would imply half-SFR sizes that are of order and often smaller than half-stellar mass sizes, suggesting that we are witnessing the build-up of central stellar bulge components. If not attributed to differences in sample selection, it may signal that tracers of dust attenuation may saturate in the centers of the most massive $z \sim 2$ SFGs. It would further imply that within a time span of merely a few hundred Myr these objects can accumulate central stellar mass densities equivalent to those observed in nearby early-type galaxies.

\section{Lessons from integral-field spectroscopy}

The three-dimensional data cubes produced by IFU spectrographs offer a wealth of information extending beyond the valuable $\mathrm{H} \alpha$ maps discussed in Section 2. Here, we focus on aspects of the internal dynamics and probes of galactic-scale feedback. The results are largely drawn from the KMOS ${ }^{3 \mathrm{D}}$ programme (PI N.M. Förster Schreiber, coPI D. Wilman), the survey design and data release of which is documented by Wisnioski et al. $(2015,2019)$. KMOS ${ }^{3 \mathrm{D}}$ combines deep integrations (from 5 hours at $z \sim 0.9$ to a median of 8.7 hours at $z \sim 2$ ) for a large number of galaxies (740 in total), spanning a wide dynamic range in star formation rate and mass, and targeting a consistent range of rest-wavelengths across the $0.6<z<2.7$ portion of cosmic history.

The mass budget of galaxies since cosmic noon. The ubiquity of ordered rotational motions observed in distant SFGs allows the gaseous kinematic moment maps to be employed to dynamically place constraints on the (total) amount of enclosed mass within the disk regions of distant galaxies. To this end, two aspects are of particular concern regarding the analysis of velocity and velocity dispersion profiles of galaxies in the early 
Universe relative to those observed nearby. First, beam smearing effects can often be severe in seeing limited observations, with a typical galaxy size to beam size ratio of 1.7 in the $\mathrm{KMOS}^{3 \mathrm{D}}$ sample. Different approaches have been employed in the literature, ranging from a simple rescaling of the galactocentric radius axis accounting for an addition of the PSF size in quadrature (Tiley et al. 2019), to the application of a lookup table with correction factors or a scaling relation based on toy model galaxies to the convert observed to intrinsic rotational velocities and derive an intrinsic velocity dispersion (e.g., Burkert et al. 2016; Johnson et al. 2018), to finally carrying out a forward modeling of the observed velocity and dispersion profiles simultaneously with rotating disk models that are mock observed under the appropriate inclination and beam smearing conditions (e.g., Cresci et al. 2009; Wuyts et al. 2016).

While beam smearing reduces the intrinsic rotational velocity leaving only an imprint in the form of an artificially enhanced observed central velocity dispersion, the presence of local random motions (often referred to as the intrinsic velocity dispersion $\sigma_{0}$ ) revealed as a plateau to the dispersion profile can on its turn impact the observed rotation curve, irrespective of beam smearing effects. Such a component of pressure support has the net effect of reducing the rotational velocity with respect to the circular velocity needed to balance a dynamically cold and hence thin disk against gravity $\left(v_{\text {rot }}^{2}=v_{\text {circ }}^{2}-2 \sigma_{0}^{2}\left(\frac{r}{R_{d}}\right)\right.$ following Burkert et al. 2010).

Accounting for both effects (beam smearing and contributions from pressure support) when modeling the dynamics of high-redshift gas-rich disks, Wuyts et al. (2016) found higher redshift SFGs, and most notably those featuring the highest surface densities, to be more baryon dominated (with $\left\langle f_{\mathrm{bar}}\left(\left\langle R_{e}\right)\right\rangle \approx 90 \%\right.$ ) than their more extended and lower redshift counterparts. A comparison to the Illustris cosmological hydro simulation and to a much simpler bath tub model in which disk growth is tied to typical halo growth histories through a set of analytical equations, illustrates that similar trends with galaxy surface density emerge naturally within a $\Lambda$ CDM context, at least at a qualitative level. Those galaxies in which the baryons managed to assemble within a compact region in the center of the parent dark matter halo naturally feature low contributions from dark matter when quantified within the disk region. Efforts to push measurements of rotation curve shapes out to more than $3 R_{e}$ through stacking (Lang et al. 2017) or yet deeper integrations (Genzel et al. 2017) confirm the baryon dominated nature of $z \sim 2$ SFGs, without reliance on assumptions and systematics that may affect the stellar and gas mass estimates in Figure $3\left(\mathrm{IMF}, \alpha_{\mathrm{CO}}, \ldots\right)$.

Returning to the reconstruction of evolutionary sequences discussed in Section 1, we recall that the bulk of $z \sim 2$ galaxies in the kinematic samples from $\operatorname{KMOS}^{3 \mathrm{D}}$ will not evolve into present-day Milky Way-mass systems but will rather end up as higher mass galaxies, predominantly featuring early-type morphologies. If we do not contrast high-z disks to typical spirals in the local Universe, but instead to their more likely early-type descendants, it is noteworthy that those too feature very low dark matter fractions within the confines of their stellar extent (Courteau \& Dutton 2015).

Equivalent comparisons between dynamical and stellar masses for galaxies that are already quiescent by cosmic noon have been pursued based on their stellar dynamics. Given their compact sizes, this is most commonly done by means of a virial mass estimator applied to the galaxy-integrated velocity dispersion. In recent years, however, it has become increasingly clear that the quiescent population cannot be treated as purely pressure supported systems, but instead also features substantial amounts of rotational support, as anticipated from a highly dissipational formation process (e.g., Robertson et al. 2006; Wuyts et al. 2010; Wellons et al. 2015). First empirical clues to this end came from a statistical analysis of the axial distribution of early quiescent galaxies, 

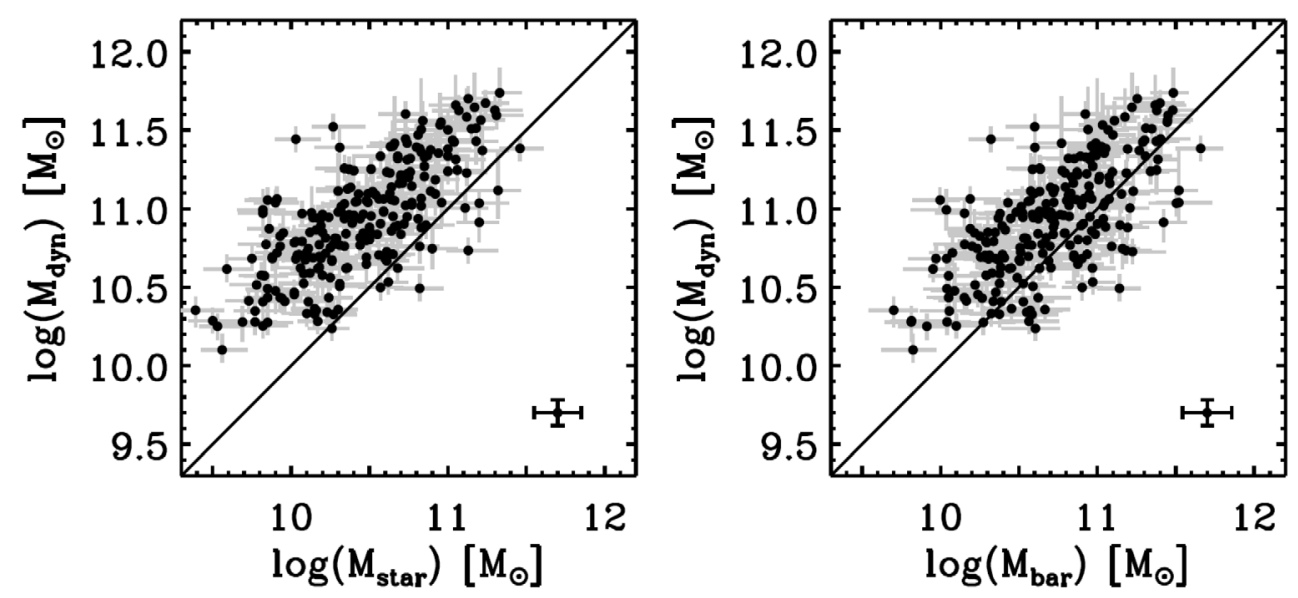

Figure 3. Dynamical mass contrasted to the stellar mass (left) and to the baryonic (i.e., stellar + gas) mass (right) for $240 \mathrm{KMOS}^{3 \mathrm{D}}$ galaxies at $0.6<z<2.6$ (reproduced from Wuyts et al. 2016). While the different mass estimates show a clear correlation, they are offset from the one-to-one line and the scatter around the relation is larger than what can be accounted for by measurement uncertainties. The considerable range in redshifts contributes to part of the scatter, with $z \gtrsim 2$ galaxies being more baryon dominated in their disk regions than their lower redshift counterparts. More predictive of the observed stellar-to-dynamical and baryonic-to-dynamical mass ratio still are measures of the galaxies' surface densities. Extended galaxies probe further into their parent halos and hence contain larger dark matter contributions within $R_{e}$.

exhibiting a larger number of elongated projected shapes relative to nearby quiescent galaxies (van der Wel et al. 2011; Chang et al. 2013). More recently, the presence of rotational motions and hence need for inclination corrections in deriving dynamical masses was alluded to based on galaxy-integrated dynamical measurements by Belli et al. (2017). Finally, owing to fortuitous lensing magnifications, Newman et al. $(2015,2018)$ and Toft et al. (2017) were able to spatially resolve the rotation curves of a few $z \sim 2$ quiescent galaxies, placing them on the upper envelope of the distribution of so-called 'fast rotators' among nearby early-type galaxies in angular momentum - ellipticity space. Bezanson et al. (2018) leverage the larger number statistics from LEGA-C to demonstrate that already at $z \sim 0.8$ a shift toward higher angular momentum quiescent galaxies is notable.

Turbulence in the ISM. Aside from its impact on the inferred dynamical mass budget and the observed rotation curve shapes at cosmic noon, the enhanced levels of turbulence in the ISM of distant galaxies, signaled by a floor to the dispersion profile and parameterized by $\sigma_{0}$, are interesting in their own right. This because it poses the question which process(es) is/are responsible to driving the turbulence and because they can potentially offer an empirical angle on the yet elusive but essential gaseous accretion flows required to continuously replenish the gas-rich high-z disks that feature relatively low depletion times $\left(t_{\text {dep }} \equiv \frac{M_{\text {gas }}}{S F R}<1\right.$ Gyr $)$.

Übler et al. (2019) present the latest compilation of intrinsic velocity dispersion measurements. In the ionized gas phase the level of turbulence progressively increases with redshift, reaching $\sigma_{0} \approx 45 \mathrm{~km} \mathrm{~s}^{-1}$ at $z \sim 2.3$, roughly twice of what is typical for nearby disks. In the cold gas phase, the number of measurements at high redshift is still modest, but early ALMA and NOEMA results suggest a similar increase with redshift, albeit offset downward by $10-15 \mathrm{~km} \mathrm{~s}^{-1}$. In other words, it is not just the ionised gas at $10^{4}$ $\mathrm{K}$ that is more easily stirred by the enhanced levels of star formation at cosmic noon. In fact, contrasting their measurements to a theoretical model by Krumholz et al. (2018) they conclude that stellar feedback alone is not capable to explain the full range of $\sigma_{0}$ 


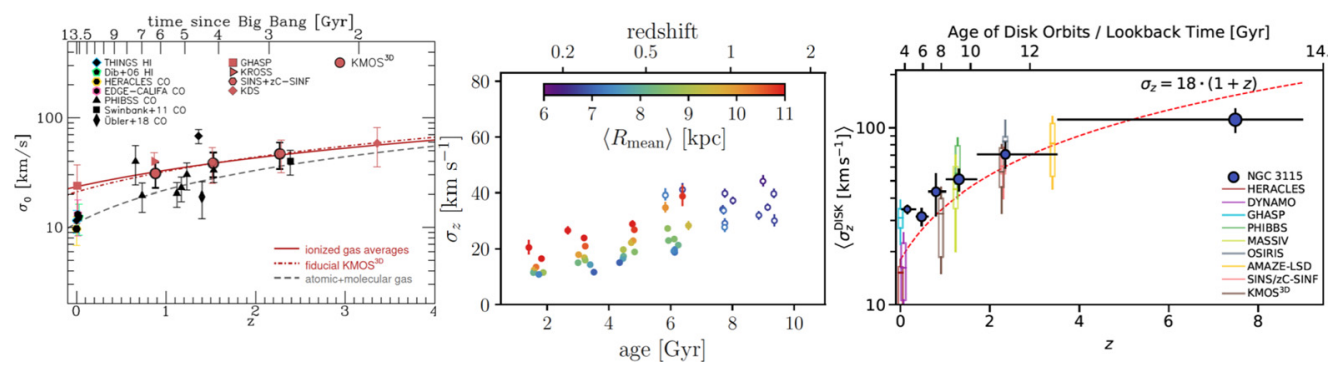

Figure 4. From left to right: Intrinsic velocity dispersion of ionized and cold gas as a function of redshift (reproduced from Übler et al. 2019); Galactic vertical velocity dispersion as a function of stellar age (reproduced from Mackereth et al. 2019); Vertical velocity dispersion as a function of age of disk orbits in NGC3115 (reproduced from Poci et al. 2019).

values observed, and specifically their relation to the galaxies' observed SFR, rotational velocity and gas fraction. Instead, a significant source of powering the turbulent velocities in the marginally stable high-z disks is attributed to gravitational instabilities.

Figure 4 illustrates that the observed increase in the intrinsic velocity dispersion with redshift is reminiscent of recent results obtained from Galactic star-by-star archeology and fossil record studies exploiting 3D imaging spectroscopy of a well-resolved individual nearby disk galaxy. In the latter two cases, older stellar populations are associated with progressively thicker disk structures, with a continuum in stellar ages correlating with the vertical velocity dispersion of the respective mono-age stellar population.

It is tempting to associate the similarity to the lookback survey results as an indication that the thicker disk structures were formed as such, from the more gas-rich and turbulent early disks, rather than formed thin and puffed up at a later stage by disturbances such as merger events. Here, again, the caveat applies that in order to consolidate such an interpretation progenitor and descendant galaxies need to be linked up properly. For the high-redshift studies, this generally requires a push to more adaptive optics assisted observations of low-mass galaxies, enabled by ERIS on VLT and in future years JWST as well as ELT.

Indirect probes of feedback: metallicity gradients. Theoretical models of galaxy formation require strong feedback to resolve the long-standing angular momentum problem (Navarro \& Steinmetz 2000), prevent the overproduction of stars and reproduce realistic quenched fractions as a function of galaxy mass and cosmic time. Seeing the imprint of such strong feedback observationally is an area where IFU spectrographs can make unique contributions. In an indirect manner, the fact that the metallicity gradients inferred from ionized gas line ratios are found to be weak, if not flat, on average for SFGs at cosmic noon suggests a continuous mixing of ISM material (Wuyts et al. 2016b; Förster Schreiber et al. 2018). In the absence of injections of energy and momentum, the inside-out growth of stellar disks combined with the chemical enrichment they bring about would otherwise soon lead to the emergence of declining radial profiles in the gas-phase metallicity. Zooming out beyond the disk regions into the Circum-Galactic Medium (CGM), sightline studies by Steidel et al. (2010) have demonstrated the presence of metals at impact parameters beyond $60 \mathrm{kpc}$. By lack of in situ enrichment so far into the halo the observed metal columns can only be explained by powerful galactic-scale outflows.

Direct probes of feedback: galactic winds. Capturing the launching of such winds can be achieved by decomposing emission line profiles into a systemic and high-velocity component (e.g., Förster Schreiber et al. 2019), a technique complementary to that tracing velocity offsets of interstellar absorption lines which shed light on the neutral phase of the outflow (e.g., Shapley et al. 2003). To this end, IFU data sets offer the unique advantage 
that the resolved velocity field can be taken out, thereby reducing the width of the systemic component and hence enhancing the contrast with the broad velocity component representing non-gravitational motions. Numbers, depth and dynamic range in galaxy properties sampled by surveys such as $\mathrm{KMOS}^{3 \mathrm{D}}$ have recently brought studies in this area to a stage where not only the occurence of this phenomenon can be established, but its demographics can be mapped across the SFR - mass plane and its energetics and scaling relations can be compared to those predicted by theoretical models. Förster Schreiber et al. (2019) make the case that the outflow phenomenology is best studied for galaxies featuring/lacking diagnostics of AGN activity separately, as their respective broad components differ in characteristic width $\left(\mathrm{FWHM} \sim 400-600 \mathrm{~km} \mathrm{~s}^{-1}\right.$ for star formation driven winds versus FWHM $\sim 1000-2500 \mathrm{~km} \mathrm{~s}^{-1}$ for those emerging from galaxies hosting AGN) and in their demographics. Star formation driven winds are most prevalent in galaxies residing above the star-forming main sequence, where the highest star formation surface densities (and hence highest energy and momentum injection rates from supernovae) are found. The AGN driven winds on the other hand show a steep mass dependence, becoming increasingly common as one considers galaxies above the Schechter mass, irrespective of their level of star formation activity. Moreover, the inferred energetics are such that much of the material launched by stellar feedback may not make it out of the parent halo and is anticipated to rain back in the form of a galactic fountain. Conversely, the strong outflows emerging from AGN-hosting galaxies at the high-mass end may do more (long-term) damage to the gas reservoirs.

\section{Outlook and open questions}

Deep multi-wavelength lookback surveys with HST mapping the rest-UV to restoptical emission from distant SFGs on kiloparsec scales, combined with integral-field spectroscopy and accumulating resolved dust continuum and CO data sets are providing a rich multi-tracer view on the nature of gas-rich disk galaxies at cosmic noon. The highly multiplexed nature of new near-infrared (and particularly IFU) spectrographs has enabled placing early results gathered painstakingly by observing of order 1 galaxy per observing night on an 8-meter telescope on a more robust statistical footing. In addition, recent results have also prompted a new array of questions, many of which are to be tackled with the next generation of instruments and observing facilities (Figure 5).

A non-exhaustive list building on the aforedescribed findings include:

- Where do massive $z \sim 2$ SFGs form their last stars before they get quenched? Balmer decrement maps for individual galaxies and bolometric UV+IR SFR maps accounting for potential gradients in dust temperature will be required to address whether halfSFR sizes at the tip of the main sequence are smaller than, equal to or larger than the half-stellar mass sizes inferred from multi-wavelength HST imagery.

- What is the origin of scatter in galaxy scaling relations $\left(S F R-M_{\mathrm{star}}, R_{e}-M_{\mathrm{star}}\right.$, $\left.Z-M_{\text {star }}, \ldots\right)$ ? Is any scatter around the observed relations attributed to short-term stochasticity (i.e., the equivalent of 'weather') or an imprint of a long-term differentiation in growth histories among SFGs of the same mass at a given epoch. If the latter, what (halo) property other than mass is most appropriate to describe the SFG population as a two-parameter family? And which observable best serves as an empirical proxy?

- What is the origin of 'unphysical' baryon fractions $\left(M_{\mathrm{bar}} / M_{\mathrm{dyn}}>1\right)$ ? A robust trend of increasing baryon fractions with increasing surface density is emerging from disk modeling of IFU kinematics. At its extremes, the number of galaxies for which the baryonic mass enclosed within $R_{e}$ exceeds the dynamical constraint $M_{\mathrm{dyn}}\left(<R_{e}\right)$ appears larger than what can be accounted for by random uncertainties. Are these compact SFGs closer to being gas depleted than conventional gas scaling relations, established for the bulk of 

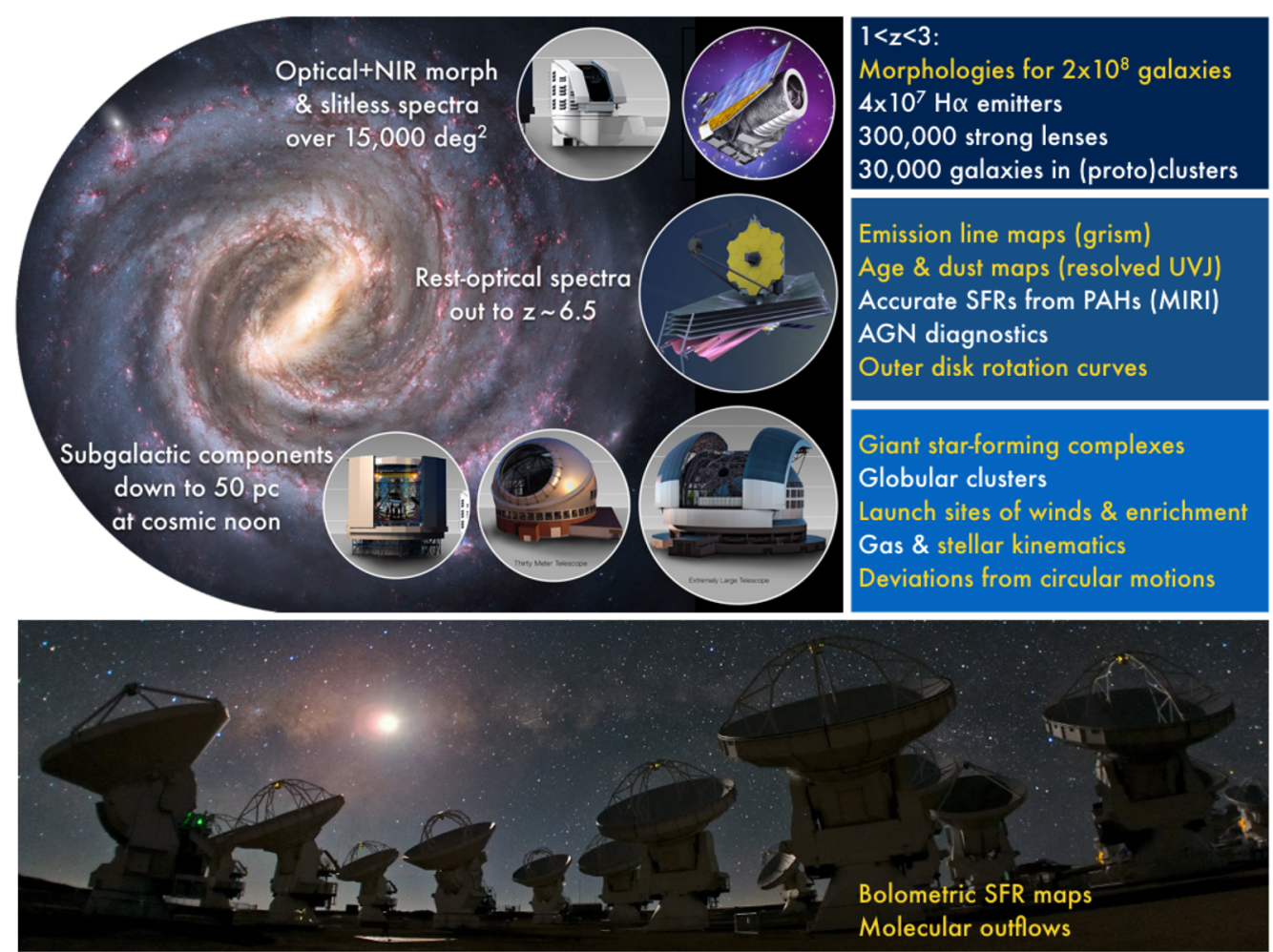

Figure 5. Top: A new generation of wide-area survey telescopes (LSST, Euclid) on the one hand, and the ultra-sensitive and high-resolution capabilities of JWST and a new class of Extremely Large Telescopes on the other hand, promises to revolutionize the study of galaxies at cosmic noon. These facilities will open a window on an array of outstanding questions inspired by the recent studies reviewed in this paper. Bottom: Complementing these rest-UV, rest-optical and near/mid-IR instruments, high-resolution observations with interferometers such as ALMA and NOEMA in the far infrared will remain indispensible to shed full light on the physics at play, by complementing stellar and ionized gas tracers with probes of dust and molecular gas emission.

the galaxy population, suggest? Are uncertainties on their dynamical masses underestimated (e.g., because in the most extreme cases axial ratio based inclinations are hard to determine accurately for marginally resolved compact nuggets)? Or do they represent a subpopulation for which treatment with thick disk models becomes inadequate?

- What is the physics responsible for setting $\sigma_{0}$ ? The redshift evolution of $\sigma_{0}$ can be understood in the framework of marginally stable disks with gas fractions that are dwindling with cosmic time (Wisnioski et al. 2015; Übler et al. 2019). Yet, at fixed redshift no statistically significant relations between $\sigma_{0}$ and for example the gas fraction within galaxies can be discerned within current data sets. Is this because the dynamic range sampled at a given epoch is modest, and measurement uncertainties in the relevant quantities $\left(\sigma_{0}, f_{\text {gas }}\right)$ comparatively large? Or are we missing physics? Furthermore, nearby galaxies are known to feature velocity anisotropies with radial velocity dispersions $\sigma_{R}$ typically exceeding those measured along the axis orthogonal to the disk plane. In contrast, the assumption of an isotropic velocity ellipsoid (i.e., $\sigma_{0}=\sigma_{R}=\sigma_{z}$ ) is commonly adopted in the analysis of high-z kinematics.

- Which processes set the local gas-phase metallicity? The largest samples of resolved (with AO) and marginally resolved (in seeing limited mode) metallicity gradients of 
SFGs at cosmic noon to date are based on the N2 (i.e., [NII]/H $\alpha$ ) diagnostic. Its known caveats leave room for improvement, e.g., by employing multi-line diagnostics calibrated for the evolving ISM conditions within distant SFGs compared to normal nearby spirals. KLEVER, a VLT Large Programme piggybacking on KMOS ${ }^{3 \mathrm{D}}$ and extending it with a sample of lensed galaxies that benefit from enhanced magnification, serves as an example where resolved $\mathrm{H} \beta$, [OIII], $\mathrm{H} \alpha$ and [NII] maps can offer a more comprehensive view on the local ISM conditions.

- What are the total mass loading and energetics of galactic-scale winds and how does it break down into multi-phase components? Much of what was discussed above in terms of wind properties and demographics was based on the ionized phase only. A more holistic view on wind properties and their impact on a galaxy's evolutionary path requires the combination of multi-phase tracers. To date, the number of individual normal main sequence SFGs (as opposed to luminous starbursting outliers or extreme quasars) with wind properties mapped in the neutral phase through interstellar absorption line shifts, as well as in the ionized and molecular phase through broad components to the [OIII] or $\mathrm{H} \alpha$ and $\mathrm{CO}$ emission lines, remains extremely small. A pilot programme by Herrera-Camus et al. (2019) suggests that, equivalent to what is seen in nearby starbursts, the bulk of the mass flow may be in the molecular phase, implying that ionized gas observations alone are insufficient to fully capture their impact on galaxy evolution.

New facilities that will come online this decade promise to advance our knowledge on most of the above outstanding questions. They will yield basic structural measurements for samples orders of magnitude larger than presently available, copious strong lenses to carry out higher resolution studies of internal galaxy structure and kinematics, and significantly enhanced sensitivities and spatial resolution to dissect the in situ physical processes within high-z disks, even for blank field galaxies that lack the systematic uncertainties associated with lens modeling (differential magnification etc.). Among many other areas, this will open a window on the fragmentation processes within marginally stable disks, on not only gas but also stellar kinematics within distant SFGs, and on anticipated deviations from circular motions enabling tests of in situ bulge formation scenarios in which radial gas flows result from violent disk instabilities.

\section{References}

Abramson, L. E., Gladders, M. D., Dressler, A., Oemler, A. Jr., Poggianti, B., \& Vulcani, B. 2016, ApJ, 832, 7

Allen, R. J., Kacprzak, G. G., Glazebrook, K., et al. 2017, ApJ, 834, 11

Belli, S., Newman, A. B., \& Ellis, R. S. 2017, ApJ, 834, 18

Bezanson, R., van der Wel, A., Pacifici, C., et al. 2018, ApJ, 858, 60

Brammer, G. B., van Dokkum, P. G., \& Coppi, P. 2008, ApJ, 686, 1503

Bullock, J. S., Dekel, A., Kolatt, T. S., et al. 2001, ApJ, 555, 240

Burkert, A., Förster Schreiber, N. M., Genzel, R., et al. 2016, ApJ, 826, 214

Burkert, A., Genzel, R., Bouché, N., et al. 2010, ApJ, 725, 2324

Chang, Y.-Y., van der Wel, A., Rix, H.-W., et al. 2013, ApJ, 762, 83

Courteau, S. \& Dutton, A. A. 2015, ApJ, 801, 20

Cresci, G., Hicks, E. K. S., Genzel, R., et al. 2009, ApJ, 697, 115

Davidzon, I., Ilbert, O., Laigle, C., et al. 2017, A\&A A, 605, 70

Förster Schreiber, N. M., Renzini, A., Mancini, C., et al. 2018, ApJS, 238, 21

Förster Schreiber, N. M., Übler, H., Davies, R. L., et al. 2019, ApJ, 875, 21

Genzel, R., Förster Schreiber, N. M., Übler, H., et al. 2017, Nature, 543, 397

Herrera-Camus, R., Tacconi, L., Genzel, R., et al. 2019, ApJ, 871, 37

Huang, K.-H., Fall, S. M., Ferguson, H. C., et al. 2017, ApJ, 838, 6

Johnson, H. L., Harrison, C. M., Swinbank, A. M., et al. 2018, MNRAS, 474, 5076

Kravtsov, A. V. 2013, ApJ, 764, 31 
Krumholz, M. R., Burkhart, B., Forbes, J. C., \& Crocker, R. M. 2018, MNRAS, 477, 2716

Lang, P., Wuyts, S., Somerville, R. S., et al. 2014, ApJ, 788, 11

Lang, P., Förster Schreiber, N. M., Genzel, R., et al. 2017, ApJ, 840, 92

Liu, F. S., Dongfei, J., Faber, S. M., et al. 2017, ApJ, 844, 2

Mackereth, J. T., Bovy, J., Leung, H. W., et al. 2019, MNRAS, in press (arXiv:1901.04502)

Matthee, J. \& Schaye, J. 2019, MNRAS, 484, 915

Poci, A., McDermid, R. M., Zhu, L., \& van de Ven, G. 2019, MNRAS, 487, 3776

Mo, H. J., Mao, S., \& White, S. D. M. 1998, MNRAS, 295, 319

Mowla, L., van der Wel, A., van Dokkum, P. G., \& Miller, T. B. 2019, ApJ, 872, 13

Navarro, J. F. \& Steinmetz, M. 2000, ApJ, 538, 477

Nelson, E. J., van Dokkum, P. G., Brammer, G., et al. 2012, ApJ, 7474, 28

Nelson, E. J., van Dokkum, P. G., Momcheva, I., et al. 2013, ApJ, 763, 16

Nelson, E. J., van Dokkum, P. G., Förster Schreiber, N. M., et al. 2016a, ApJ, 828, 27

Nelson, E. J., van Dokkum, P. G., Momcheva, I., et al. 2016b, ApJ, 817, 9

Newman, A. B., Belli, S., \& Ellis, R. S. 2015, ApJ, 813, 7

Newman, A. B., Belli, S., Ellis, R. S., \& Patel, S. G. 2018, ApJ, 862, 126

Ribeiro, B., Le Fèvre, O., Tasca, L. A. M., et al. 2016, A\&A, 593, 22

Robertson, B., Bullock, J. S., Cox, T. J., et al. 2006, ApJ, 645, 986

Sanders, R. L., Shapley, A. E., Kriek, M., et al. 2018, ApJ, 858, 99

Shapley, A. E., Steidel, C. C., Pettini, M., \& Adelberger, K. L. 2003, ApJ, 588, 65

Speagle, J. S., Steinhardt, C. L., Capak, P. L., \& Silverman, J. D. 2014, ApJS, 214, 15

Steidel, C. C., Erb, D. K., Shapley, A. E., et al. 2010, ApJ, 717, 289

Suess, K. A., Kriek, M., Price, S., \& Barro, G. 2019, ApJ, 877, 103

Tacchella, S., Carollo, C. M., Förster Schreiber, N. M., et al. 2018, ApJ, 859, 56

Tacconi, L. J., Genzel, R., Saintonge, A., et al. 2018, ApJ, 853, 179

Tadaki, K., Genzel, R., Kodama, T., et al. 2017, ApJ, 834, 135

Tiley, A. L., Swinbank, A. M., Harrison, C. M., et al. 2019, MNRAS, 485, 934

Toft, S., Zabl, J., Richard, J., et al. 2017, Nature, 546, 510

Torrey, P., Wellons, S., Ma, C.-P., Hopkins, P. F., \& Vogelsberger, M. 2017, MNRAS, 467, 4872

Übler, H., Förster Schreiber, N. M., Genzel, R., et al. 2017, ApJ, 842, 121

Übler, H., Genzel, R., Wisnioski, E., et al. 2019, ApJ, 880, 48

van der Wel, A., Rix, H.-W., Wuyts, S., et al. 2011, ApJ, 730, 38

van der Wel, A., Franx, M., van Dokkum, P. G., et al. 2014, ApJ, 788, 28

Wellons, S., Torrey, P., Ma, C.-P., et al. 2015, MNRAS, 449, 361

Whitaker, K. E., Franx, M., Bezanson, R., et al. 2015, ApJ, 811, 12

Wilman, D. J., Fossati, M., Mendel, J. T., et al. 2019, ApJ, submitted

Wisnioski, E., Förster Schreiber, N. M., Wuyts, S., et al. 2015, ApJ, 799, 209

Wisnioski, E., Förster Schreiber, N. M., Fossati, M., et al. 2019, ApJ, submitted

Wuyts, S., Cox, T. J., Hayward, C., et al. 2010, ApJ, 722, 1666

Wuyts, S., Förster Schreiber, N. M., van der Wel, A., et al. 2011, ApJ, 742, 96

Wuyts, S., Förster Schreiber, N. M., Genzel, R., et al. 2012, ApJ, 753, 114

Wuyts, S., Förster Schreiber, N. M., Nelson, E. J., et al. 2013, ApJ, 779, 135

Wuyts, S., Förster Schreiber, N. M., Wisnioski, E., et al. 2016, ApJ, 831, 149

Wuyts, E., Wisnioski, E., Fossati, M., et al. 2016, ApJ, 827, 74

Zanisi, L., Shankar, F., Lapi, A., et al. 2019, MNRAS, submitted 